\section{A REVIEW OF THE ANTIOXIDANTS FROIM THE MEDICINAL PLANT, MORINGA OLEIFERA}

KEY WORDS: Moringa oleifera, medicinal plant, antioxidant properties, phenolics, flavonoids, seed oil.

\section{Sai Janani K}

Department of botany, 36, lalbagh road, st. Joseph's college (autonomous), Bangalore-560027.

Department of botany, 36, lalbagh road, st. Joseph's college (autonomous), Bangalore- 560027. ${ }^{*}$ Corresponding Author

Moringa oleifera, is a highly potent medicinal plant which is evergreen or deciduous in nature and it is found growing predominantly in the Sub-Himalayan regions of India, Pakistan, Bangladesh and Afghanistan. This plant is tagged as a medicinal plant because it contains various properties such as high antioxidant properties, anti- inflammatory, anticancer, antidiabetic and antimicrobial properties. This review paper will highlight, focus and discuss the antioxidant properties possessed by Moringa oleifera supported by some experiments containing materials required, methods employed, results procured and discussion to support the evidences that prove the antioxidant properties found in this plant. The first and the second experiment is about the presence of flavonoid contents like Quercetin and Rutin found respectively that contributes to the antioxidant properties found in the plant of subject, Moringa oleifera. While, the third experiment is based on the application of the antioxidant properties found in the plant that contributes towards the enhancement of the meat quality thereby, this attribute of the plant having antioxidant properties is used in the meat industries.

\section{INTRODUCTION}

Moringa oleifera (Fig-1), a vital medicinal plant is one of the most extensively cultivated species of the family Moringaceae and Sub-Himalayan tracts of India, Pakistan, Bangladesh and Afghanistan is found to be its native [1]. This plant is a small, evergreen, or deciduous, fast-growing tree that usually grows to a height of 10 or $12 \mathrm{~m}$ [2]. Its stem and drooping branches are brittle, with corky bark. Pale -green, feathery, compound, tripinnate leaves is observed; white or creamy-white fragrant flowers are present with diameter $2.5 \mathrm{~cm}$ which has the presence of yellow colour stamens; pods that are tapered on both the ends are triangular, pendulous and brown in colour containing nearly 20 seeds that are dark brown embedded within the pith [3]. This plant has got medicinal properties to treat anxiety, asthma, bronchitis, chest congestion and cough, tuberculosis cholera, diarrhoea, eye and ear infections, conjunctivitis, fever, anaemia, abnormal blood pressure and blood impurities, scurvy, pain in joints, semen deficiency etc [2]. The leaves, pods and seeds of Moringa oleifera has immense nutrition due to the presence of various essential phytochemicals. Apparently, vitamin $\mathbf{C}$ content in Moringa is 7 times more than that of oranges, vitamin A content is 10 times more than carrots, protein content is 9 times more than yoghurt, 17 times more calcium content than milk, source of potassium 15 times more than bananas and has 25 times more iron content than spinach [4]. Also, this medicinal plant is a potential antioxidant, anticancer, anti-inflammatory, antidiabetic and antimicrobial agent [5]. As mentioned above, this plant is a potential antioxidant agent that prevents free radical production that occurs in all cells performing cellular functions leading to tissue damage [6]. Oxidative stress is caused by free radical molecules due to disproportion between the reactive oxygen species (ROS) and the antioxidant defence system. Oxidative damage is caused by the activated ROS to the biomolecules like proteins, lipoproteins lipid and DNA which are considered to be essential [7]. As a result of this diseases like cancer, diabetes, aging, liver cirrhosis and Alzheimer's disease is caused [8].

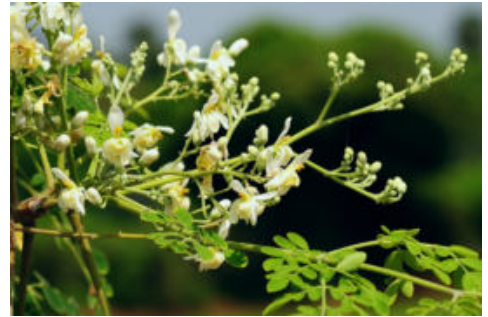

Fig-1: Moringa oleifera plant showing flowers. Image courtesy [28]

\section{MATERIALS AND METHODS}

(1)antioxidant Properties Witnessed In Moringa Oleifera Leaves

Chemicals used: Folin-Ciocalteu's phenol reagent, deionized water, Ethanol, Aluminium Chloride, Potassium acetate, Methanol and Dichloromethane.

\section{Plant and extraction:}

The collected air-dried leaves of Moringa oleifera is grounded into powder form and further it is stored at $4^{\circ} \mathrm{C}$. Methanol of $350 \mathrm{ml}$ is added to $15 \mathrm{~g}$ of air-dried leaf powder of $M$. oleifera. The air-dried leaf powder and methanol extract is filtered using Whatman no.l filter paper. Dichloromethane of $350 \mathrm{ml}$ is added to the residue for the extract. The two crude extracts, methanol extract (ME) and dichloromethane extracts (DE)are obtained from the filtrates that is evaporated and lyophilized. Until usage, both the extracts are stored in amber glass at $-20^{\circ} \mathrm{C}$. [9]

\section{Determination of total amount of flavonoid and phenolic contents -}

In order to determine the total amount of phenolic compound, the Folin - Ciocalteu method is used where $2 \mathrm{ml}$ of $50 \%$ FolinCiocalteu's phenol reagent and $2.8 \mathrm{ml}$ of deionized water is mixed with each of the diluted extracts [ (ME) and (DE)] each measuring $100 \mu l$ and incubated at room temperature for 30 minutes. The rection mixture recorded the absorbance at 765 $\mathrm{nm}$ (nanometre). The expression for the total amount of phenolic content is milligram Gallic acid equivalent per gram extract (mg GAE/g extract). [10]

In order to determine the total amount of flavonoid content, the Aluminium Chloride Colorimetric method is employed in which $100 \mu \mathrm{l}$ of $10 \% \mathrm{AlCl}_{3}, 1 \mathrm{M}$ of Potassium Acetate measuring $100 \mu \mathrm{l}$, deionized water of $2.8 \mathrm{ml}$ measurement and $95 \%$ of Ethanol measuring $1.5 \mathrm{ml}$ is added to each of the extracts measuring $100 \mu \mathrm{l}$. The reaction mixture recorded the absorbance as $415 \mathrm{~nm}$ (Nanometre). The expression for the total flavonoid content is Quercetin equivalent per gram extract (mg QE/g extract).[11]

\section{(2) Antioxidant Properties Found In Moringa Oleifera Seed Oil}

Chemicals used: $\mathrm{n}$ - hexane, methanol, sodium nitrate, aluminium trichloride,sodium hydroxide.

Extraction of oil from Moringa oleifera seeds: Husk removed whole seeds of Moringa oleifera weighing $123.49 \mathrm{~g}$ is taken 
and seeds weighing $86.7 \mathrm{lg}$ is crushed using mortar and pestle. Using the Soxhlet extraction method, oil is extracted from the crushed seeds using $\mathrm{n}$ - hexane measuring $0.5 \mathrm{~L}$ which acts like a solvent of extraction that is evaporated for 12 hours or completely by using a water bath.[12]

Determination OfThe Total Amount Of Flavonoid Content Aluminium trichloride colorimetric method where rutin is taken as standard is used to determine the total amount of flavonoid present in the oil sample by forming flavonoid aluminium complex. The seed oil sample of $0.1 \mathrm{~m}$ in $100 \mu \mathrm{l}$ of methanol is taken and added with $5 \%$ Sodium nitrate measuring $0.2-1 \mathrm{ml}$ and allowed to react for few minutes say 5 minutes. After which $0.2 \mathrm{ml}$ of Aluminium trichloride in 10\% methanol and $1 \mathrm{M}$ Sodium Hydroxide measuring $\mathrm{l} \mathrm{ml}$ is added and allowed to react in room temperature for about 15 minutes. The absorbance recorded was $510 \mathrm{~nm}$ against the blank reagent. Using rutin calibration curve, the total amount of flavonoid is estimated and expressed as $\mathrm{mg}$ of rutin equivalent per gram of sample ( $\left.\mathrm{mg} \mathrm{RE} \mathrm{g}^{-1}\right)$. [13]

\section{(3) The Role Of Natural Antioxidants Present In Moringa Oleifera That Contributes To The Enhancement Of Meat Quality}

Xhosa goats with lop ears which is castrated (removal of testicles) having an average body mass of about $14.63 \pm 0.26$ $\mathrm{kg}$ is used as the experimental animals for this particular experimental study. To avoid external parasitic infection the goats are administered with Drastic deadline [ flumethrin $1 \%$, Bayer (Pty) Ltd Isando, South Africa]. For the sake of adaptation to the feeding methods and the environment of the housing, the goats were kept in the environmental set-up for a period of 21 days. The goats were divided into three groups, each group containing eight goats. All the three groups of goats were fed with a basal diet containing Grass Hay (GH) and wheat bran (200g per head per day) and water as per needed or desired. Moringa oleifera leaves (MOL) were supplemented to the first group of goats and Sunflower cake (SC) were supplemented for the second group of goats. Equal quantity of supplements was fed to the goats two times a day (08:00 $\mathrm{h}$ and 15:00 $\mathrm{h}$ ). The second group animals being the positive control group was fed with Sunflower cake (SC) containing $170 \mathrm{~g}$ per head per day which was the source of protein. While, the first group of goats which is known to be the experimental group was fed with the source of protein contained in the leaves of Moringa oleifera (MOL) containing $200 \mathrm{~g}$ per head per day. From the beginning of the experiment, the supplementary diets [the Sunflower cake (SC) and the Moringa oleifera leaves (MOL)] were made to be isonitrogenous and isocaloric. For a time period of 60 days, the amount of energy and protein needed for the goats was measured to be 6.4 MJ ME and 74.3g CP per day. The goats were sympathetically slaughtered and the process of evisceration was performed as soon as the slaughter was performed. A 10\% weight in volume of liver is cut as soon as the slaughter was completed and was homogenized using a Phosphate buffer $(0.001 \mathrm{M})$ having a $\mathrm{pH}$ of 7. [14]

\section{Lipid Peroxidation Estimation -}

Colorimetric methods were employed to determine the Lipid peroxidation in the liver by using the Thiobarbituric acid reactive substances (TBARS) using Malondialdehyde (MDA) as a standard. $10 \%$ weight in volume of homogenized liver of $0.1 \mathrm{~mL}$ is treated with $1: 1: 1$ ratio of TBA-TCA-HCl $(0.37 \%$ of Thiobarbituric acid, Trichloroacetic acid of $15 \%$ and $\mathrm{HCl}$ of $0.25 \mathrm{~N}$ ) reagent measuring $2 \mathrm{~mL}$. The test tubes are cooled after being exposed to boiling water bath for a time period of 30 minutes. By using the Spectrophotometer for a time period of 5 minutes at intervals of 1 minute against the blank solution which is a reference, the measurement of clear supernatant's absorbance at $535 \mathrm{~nm}$ (nanometre) is measured and by performing this step we can determine the amount or quantity of Malondialdehyde found in each of the test tubes containing the samples.
With the help of the equation: \% inhibition of lipid peroxide= $\left\{A_{\circ}-A_{1}\right\} / A_{\circ} \times 100$, in which $A_{\circ}$ stands for Absorbance of the control and $A_{1}$ stands for Absorbance of the extracted sample, we can determine the Percentage activity.[14]

\section{RESULTS AND DISCUSSION}

Through Folin-Ciocalteau method it was determined that the extracts of the leaves of the medicinal plant Moringa oleifera has the presence of Phenolic contents. By performing the method, it is said that Methanol extracts (ME) had higher content (216.45 $44.64 \mathrm{mg} \mathrm{GAE} / \mathrm{g}$ extract) when compared to Dichloromethane extracts (DE) (100.12 $33.70 \mathrm{mg} \mathrm{GAE} / \mathrm{g}$ extract).[9] By using the Aluminium chloride Colorimetric method, it was inferred that the leaf extracts of Moringa oleifera has the presence of Flavonoid contents. Methanol extracts (ME) measured 65.38 $\pm 2.37 \mathrm{mg} \mathrm{QE} / \mathrm{g}$ extract which is considered to be higher than Dichloromethane extracts (DE) that measured $40.14 \pm 3.31 \mathrm{mg} Q E / g$ extract.[9] Further, by performing Aluminium chloride Colorimetric method, it was observed that the Moringa oleifera seed oil sample also had the presence Flavonoids. The Moringa oleifera seed oil samples measured $18.24 \pm 0.01 \mathrm{mg} \mathrm{RE} \mathrm{g}^{-1}$ extract [12]. There is a spike in recognising, researching and enhancing the plant products that is advantageous to humankind. Moringa oleifera belonging to the family Moringaceae is a plant with immense health, nutritional and economic abilities [12]. It is said that phenolic compounds are found in abundance in the plant parts especially the pollens, flowering tissues and leaves [15]. It is the phenolic compounds also known as the plant secondary metabolites that aids the plants in its normal development and growth, defence mechanisms counteracting the pathogens and parasites that causes infections and in generation of free radicals [16]. Phenolic compounds belong to antioxidant agents that are free radical terminators and are also a part in the process of retardation of oxidative degradation of lipids [17]. DNA mutation is the main factor that initiates the process of carcinogenesis and DNA mutation is caused by free radicals [18]. Endogenous antioxidant system prevents or hinders the process of formation of free radicals in our body with the aid of Exogenous antioxidants found in natural sources [19]. Polyphenols and flavonoids are immense in presence in the Moringa oleifera leaves and exhibit antioxidant activity [20][21]. And by performing experiments we can conclude that methanol and dichloromethane Moringa oleifera leaves extracts showed the presence phenolic contents and flavonoids and analysing the experiment we can conclude that Moringa oleifera leaves extracts with methanol was said to have more content of polyphenols like gallic acid (phenolic compound) and quercetin (flavonoid) than the Moringa oleifera leaves extracts with dichloromethane thereby, showing antioxidant activity. We can conclude by stating that due to properties like immense antioxidant activity, high potent cancer cell antiproliferation and quinone reductase induction found in Moringa oleifera extracts, it can be used in chemotherapy and chemoprevention because of its medicinal properties. [22][9]

Moringa oleifera seed oil has scarce free fatty acid composition in comparison with palm oil and also, due to this property of Moringa oleifera seed oil, it is less prone to rancidity [23]. Due to its lower acidity nature, physicochemical nature and antioxidant nature, Moringa oleifera seed oil is less economic, highly nutritional and more importantly the oil is edible in nature. Moringa oleifera seed oil has high content of oleic acid, unsaturated and low peroxide nature because of which it is used in industries like food, biofuel generation, agrochemicals, paint and varnishes manufacturing industries [12] [24] [25]. The Moringa seed oil has the presence of phenolic compounds and flavonoids which makes the oil less economic and healthy. The presence of flavonoids in the seed oil of Moringa is responsible for antioxidant action by which it performs the process of scavenging as a result of which it has the calibre to prevent cancer, heart related diseases and other chronic diseases [26]. 
By performing the Colorimetric method to determine the Lipid peroxidation in the liver of the goats that were fed with supplementary diet [ Moringa oleifera leaves (MOL) and Sunflower Cake (SC) and Grass Hay (GH)], it was observed by conducting this experiment, the percentage of lipid peroxidation in the liver of the goats supplemented with Moringa oleifera leaves (MOL) was found to be $81.33 \pm 0.99$, $38.76 \pm 0.45$ in the liver of the goats which were feed with Sunflower Cake (SC) and $1.99 \pm 0.02$ in the liver of the goats which were fed with Grass Hay (GH) [control] . These values of percentage of Lipid Peroxidation in the liver of the goats which were supplemented with MOL, SC and GH contributes to the Antioxidant activities. When we consider the percentage values of the Lipid Peroxidation in the liver of goats when supplemented with Moringa oleifera leaves (MOL) is higher than the percentage values of Lipid Peroxidation in the liver of goats which are supplemented with Sunflower Cake (SC) and Grass Hay (GH) where, the percentage value of Lipid Peroxidation in the liver of the goats which are supplemented with Grass Hay (GH) is the lowest. Moringa oleifera leaves (MOL) prevented the amount or quantity of Malondialdehyde (MDA) production in the liver of the goats that were fed with MOL [14]. As a result of this, the process of chemical spoilage caused because of the production of Reactive Oxygen Species (ROS) like singlet oxygen, peroxyl, hydroxyl radicals and superoxide anions causing Lipid Peroxidation of fats in the meat is reduced. Due to which the meat is not loaded with peroxides that causes rancid flavours in the meat and the meat's colour is also not changed [15] [27]. This leads to the employment of naturally occurring sources of Antioxidants to enhance the texture, flavour, colour, nutritional potential and the shelf life of the meat of animals as the Antioxidants stops the uncontrolled production of free radicals and species that are activated by chelating their catalytic metal ions. [7]

\section{REFERENCES:}

[1] Saurabh Dixit, A. T. (2016). Medicinal Properties of Moringa oleifera:A Review. International Journal of Education and Science Research Review, 3(2), 173-185.

[2] Garima Mishra, P. S. (2011). Traditional uses, phytochemistry and pharmacological properties of Moringa oleifera plant:an overview. Der Pharmacia Lettre Scholars Research Library,3(2), 141-164.

[3] James AD. Handbook of Energy Crops.New York. 1983

[4] J.L Rockwood, B. A. (2013). Potential uses of Moringa oleifera and an examination of antibiotic efficacy conferred by M. oleiferaseed and leaf extracts using extraction techniques available to underserved indigenous populations.Int.J.Phytotherapy Res, 3,61-71.

[5] Lakshmipriya Gopalakrishnan, K. D. (2016). Moringa oleifera: A review on nutritive importance and its medicinal application. Food Science and Human Wellness, 5, 49-56.

[6] ISYoung,J.V.(2001).Antioxidants in health and disease.JClin Pathol, 54, 176-186.

[1] Yazdanparast, R. a. (2007). In vitro Antioxidant and free radical scavenging activity of Cyperus rotundus.Journal of Medicinal Food, 10, 667-674.

[8] Adedapo, A.J. (2008). Antibacterial and antioxidant properties of the methanol extracts of the leaves and stems of Calpurnia aurea. Complemantary and Alternative Medicine, 8,53

[9] Charoensin, S. (2014). Antioxidant and anticancer activities of Moringa oleifera leaves.Academic Journals -Journal of Medicinal Plant Research, 8(7), 318-325.

[10] Singleton VL, O. R.-R. (1999). Analysis of total phenols and other oxidation substrates and antioxidants by means Folin- Ciocalteu reagent. Methods Enzymol,299, 152-178.

[11] Chang C,Y. M. (2002). Estimation of total flavonoid content in propolis by two complementary colorimetric methods.J.Food Drug Anal, 10, 178-182.

[12] H.A Ogbunugafor, F. E. (2011). Physico- chemical and antioxidant properties of Moringa oleifera seed oil. Pakistan Journal of Nutrition, 10(5), 409-414.

[13] Nile, S. a. (2010). Antioxidant activity and Flavonoid derivatives of Plumbago zeylanica.J.Nat.Products, 3, 130-133.

[14] B. Moyo, S. O. (2012). Polyphenolic content and antioxidant propperties of Moringa oleifera leaf extracts and enzymatic activity of liver from goats supplemented with Moringa oleiferal leaves / Sunflower seed cake. Elsevier Meatscience, 91,441-447.

[15] Siddhuraju, P. \&. (2003). Antioxidant properties of various solvent extracts of total pehnolic constituents from three different agroclimatic origins of drumstick tree (Moringa oleifera Lam) leaves.Journal of Agricultural and Food Chemistry, 51,2144-2155.

[16] Maisuthisakul,P.S. (2007). Assessment of of phenolic content and free- radical scavenging capacity of some Thai indigenous plants. Food Chemistry, 100, 1409-1418.

[17] Pourmorad,F.H. (2006).Antioxidant activity, phenol and flavonoid contents of some selected Iranian medicinal plants. African Journal of Biotechnology, 5,1142-1145.

[18] Johnson IT. (2004). New approaches to the role of diet in the prevention of the alimentary tract.Mutat.Res, 551(1-2), 9-28.

[19] Johnson IT. (2007). Phytochemicals and Cancer.Proc. Nutr.Soc., 66(2), 207-215.

[20] Luqman S, S. S. (2012). Experimental Assessment of Moringa oleifera leaf and fruit for its antistress, antioxidanr, and scavenging potential using in vitro and in vivo assays. Evud. Based Complement. Alternat.Med., 2012,1-12.

[21] Santos AF,A.A. (2012). Antioxidant activity of Moringa oleifera tissue extracts.

\section{Phytother.Res, 26(9), 1366-1370.}

[22] Sreelatha S,J.A. (2011). Antiproliferation and induction of apoptosis by Moringa oleifera extract on human cancer cells. Food Chem. Toxicol, 49(6), 1270-1275.

[23] Li, S. ,. (2007). A novel method for the determination of of acid value of vegetable oils. Eur.J.Lipid Sci.Tech., 109, 1088-1094.

[24] Anwar, F. a. (2007). Physico-chemical characteristics of Moringa oleifera seeds and seed oil from wild provenance of Pakistan.Pak.J.Bot., 39, 1443-1453.

[25] Manzoor, M. F. (2007). Physico - chemical characterization of Moringa concanensis seeds and seed oil.J.Am. Oil Chem.Soc, 84, 413-419.

[26] Middleton, E. J. (2000). The effects of plant flavonoids on mammalian cells: implications for immflammation, heart disease, cancer.Pharmacol.Rev, 52,673-751.

[27] Aquil, F. A. (2006). Antioxidant and free radical scavenging properties of twelve traditional used Indian medicinal plants. Turkish Journal of Biology, 30, 177-183.

[28] By Venkatx5 - Own work, CC BY-SA 3.0, https://commons. wikimedia. org/w/ index. php?curid $=20134455$ 